\title{
estudes semiótices
}

\section{Logique de l'information et sémiotique de la culture*}

\section{Anna Maria Lorusso **}

Résumé : Qu'est-ce que la post-vérité ? Qu'est-ce que les fake news ? S'agirait-il de simples pathologies de l'information ou plutôt d'un phénomène dévoilant une autre logique de la signification sociale ? En mettant à contribution les outils de la sémiotique de la culture (notamment les sémiotiques d'Umberto Eco et de Juri Lotman), le présent article entend analyser la façon dont s'est radicalement transformé le critère de la vérité des discours (leur « régime de vérité » comme le disait Foucault), de même que l'avènement d'un régime " confusionnel » se réclamant d'un critère d'authenticité fondé sur l'expérience en lieu et place d'un critère de vérité fondé sur la vérification.

Mots-clés : information ; post-vérité ; fake news ; réalisme ; authenticité.

\footnotetext{
* DOI: https://doi.org/10.11606/issn.1980-4016.esse.2021.188606 .

** Professoressa associata, Università di Bologna, Italia - Dipartimento delle Arti. E-mail: annamaria.lorusso@unibo.it.ORCID: https://orcid.org/0000-0002-2813-6511.
} 


\section{Introduction}

L e sujet de la post-vérité est à l'ordre du jour depuis maintenant plusieurs années. Tout a commencé en 2016 (avec l'élection de Trump aux ÉtatsUnis et le référendum sur le Brexit au Royaume-Uni) ; le terme de postvérité est alors entré dans le vocabulaire avec une telle force que début 2017 les dictionnaires Oxford l'ont reconnu comme le mot de l'année 2016. La centralité du sujet s'est poursuivie avec l'émergence des fake news, puis l'infodémie, en parallèle avec la pandémie.

Ces trois phénomènes - post-verité, fake news et infodémie - ont été principalement traités comme des questions d'information et de communication, mais ce sont aussi des question sémiotiques centrales, en particulier dans le cadre d'une sémiotique des cultures. Et ce n'est pas seulement parce que l'information et la communication sont en elles-mêmes des activités sémiotiques (et que par conséquent, ce qui les concerne, concerne également la sémiotique), mais surtout parce que dans la post-vérité, les fake news et l'infodémie, ce qui est en jeu est un problème de logique de la culture (dans le cadre des théories d'Eco et de Lotman), ainsi qu'un problème de régime discursif(dans le cadre de la théorie de Foucault).

Clarifions donc ce que nous entendons par ces deux termes: "logique de la culture » et « régime ».

\section{Les « logiques » de la sémiotique de la culture}

Eco se réfère à la « logique de la culture » dans son Trattato di semiotica generale (1975) ${ }^{1}$.

Dans l'Introduction, où il parle pour la première fois en termes de « logique de la culture », les deux axes de la réflexion sémiotique - la signification et la communication - sont aussitôt décrits : " il y a un système de signification (et donc un code) lorsqu'il existe une possibilité socialement acceptée de générer des fonctions de signes » et, inversement, il y a « un processus de communication lorsque les possibilités offertes par un système de signification sont exploitées par un système de communication ». "Les possibilités offertes par un système de signification » sont donc exploitées pour produire physiquement des expressions à des fins pratiques diverses.

Ces deux « espaces » de la sémiotique doivent être pris en considération ensemble, parce qu'ils sont tous les deux informés par une logique, une rationalité, qui est sémiotique: les conventions, c'est-à-dire les codes, les

${ }^{1}$ Le livre a eu seulement une traduction partielle en français : La Production des signes, Paris, Livre de Poche, 1992. C'est pourquoi je citerai l'édition italienne, en en traduisant moi-même certains passages. 
systèmes de signification ne sont pas personnels et idiosyncrasiques; il en va de même pour les processus discursifs (fondés sur ces codes), bien que dans leur variété ils présentent au niveau sémiotique des régularités de genre, de grammaire, de rhétorique, de modes de production (comme on le voit dans la dernière partie du Trattato di semiotica generale).

Ce n'est qu'en étudiant la culture comme un phénomène de communication fondé sur des systèmes de signification que nous pourrons comprendre ses mécanismes fondamentaux.

L'idée centrale est que la culture dans son ensemble obéit à des "lois sémiotiques » (Eco 1975, p. 57), et que ces lois ne peuvent être retracées qu'en reliant la question de la signification à celle de la communication.

À mes yeux, il s'agit là de l'un des points qu'il est important de souligner aujourd'hui : si nous étudions les formes fallacieuses et peu fiables d'information journalistique uniquement comme un problème de communication, nous n'obtiendrons pas une réelle compréhension du problème. Notre objectif doit être de nous concentrer sur les modes de signification sous-jacents, c'est-à-dire sur la manière dont la triangulation sémiotique se produit dans cette phase particulière de la communication contemporaine.

J'entends par triangulation sémiotique la façon dont nous pensons la relation entre l'objet, le signe et les interprétants (pour utiliser les termes de Peirce), c'est-à-dire la traduction particulière de cette tripartition entre le monde, les textes et ses interprétations. II me semble que, pour comprendre la logique de la post-vérité en tant que logique de la culture, nous devons examiner ces termes et, en particulier, la façon dont le concept de « réalité » a changé.

De fait, étudier le phénomène de la post-vérité implique une réflexion sur ce que nous entendons aujourd'hui par " réalisme » et par " discours réaliste », car ce c'est seulement ainsi que nous pourrons mieux comprendre pourquoi, aujourd'hui, des discours contradictoires ou rapidement réfutés nous paraissent tout de même crédibles.

\section{Comment les médias ont changé le sens de la réalité}

Ce qui me paraît essentiel pour comprendre la logique actuelle de la postvérité et l'idée de réalité qu'elle présuppose, c'est la confusion entre fiction et description, invention et documentation, que les médias (à commencer par les médias traditionnels : la télévision) ont générée (voir Lorusso, 2018).

J'ai l'impression qu'au cours des dernières décennies nous avons assisté à un lent processus d'hybridation qui, en mélangeant les genres télévisuels (divertissement et information, spectacle et débat) nous a habitués à des processus de communication dans lesquels il n'y a plus de véritable séparation 
entre le monde des simulacres télévisuels et le monde de la réalité. Lorsqu'elle a fait son entrée dans la télévision (dans le cadre des émissions d'investigation, de débat politique, de dénonciation...), la réalité est devenue un objet de spectacularisation, de projection émotionnelle, de participation. Nous, spectateurs et citoyens, avec toute notre " banalité », avons eu le droit de passer à la télévision (d’abord comme invités, dans les émissions des années 80, puis comme protagonistes, dans les reality shows des années 2000), de sorte que la frontière qui séparait l'expérience spontanée de la mise en scène s'est progressivement effacée.

En voulant devenir une "fenêtre sur le monde ", les émissions des années 80 (dans une phase qui, en Italie, a été définie comme "TV vérité ») ont commencé à créer des mondes in vitro. Au lieu de cadrer le réel, de le découper, de le sélectionner et de discuter des éléments d'intérêt, la télévision a commencé à construire des événements: nous sommes passés d'une vérité télévisuelle en tant que miroir de la réalité, à une télévision productrice de réalité.

La réalité médiatique qui constitue notre monde d'aujourd'hui est une sorte d'hyper-réalité : une observation en direct, au microscope, d'un cas exemplaire, d'une histoire singulière et très particulière, et c'est justement sur cette exaltation du quotidien de la vie de n'importe qui " comme si c'était la nôtre » que s'est joué le passage à la « reality-tv », nouvelle déclinaison de la tv-vérité : un tournant de la télévision vers la vérité du vécu, entre psychocomportementalisme et voyeurisme.

Le plan de réalité et les logiques de vérité que les transmissions des années 1990 et 2000 ont construit sont intrinsèquement complexes. La réalité qu'un reality-show ou un contest-show montre est vraie en ce qui concerne la correspondance avec les faits (les faits se passent effectivement comme nous les voyons), mais pourrait-on en dire autant de la plausibilité des faits (est-il normal que des dynamiques comme celles-là se produisent)? Et que dire de la " naturalité », la spontanéité, que nous voyons, et qui pose la question de la façon dont le cours des événements est conditionné par le système médiatique ? Dans ces cas, la télévision ne fonctionne pas tant comme une fenêtre sur le monde que comme un miroir déformé de celui-ci : un écran sur lequel nous voyons projetés des « egos » possibles, avec des passions et des émotions qui peuvent être les nôtres.

En effet, la vérité des reality-shows se transmet avant tout sur un plan émotionnel : les situations mises en scène sont forcées (pas fausses) car le cadre expérientiel établi à l'avance est lui-même forcé, mais ce qui s'y passe est authentique: cela répond à des impulsions, à des actions-réactions, vraies. 
De la vérité des contextes (le contexte du monde réel qui est mis au premier plan par de nombreuses émissions), nous sommes passés à la vérité des sentiments et des expériences vécues.

\section{De la véridiction à l'authentification}

Je n'emploie pas le mot « expérience » par hasard, car je pense que la façon dont nous comprenons l'expérience aujourd'hui joue un rôle fondamental dans nos codes de signification (et donc dans la logique de la culture qui régit nos processus de communication aujourd'hui: post-vérité, fausses nouvelles, infodémie...).

De nos jours, l'expérience me paraît le paramètre de légitimation fondamentale du discours ; l'interprétant typique qui unit le signe et l'objet, ou la médiation interprétative qui légitime la pertinence d'un certain discours par rapport à un certain objet. Pensons aux nombreux discours anti-vaccins qui circulent : ils font très souvent référence à des expériences privées de " dégâts » causés par un médicament ou par un vaccin (le père qui dit avoir perdu son fils alors que celui-ci venait de se faire vacciner; le jeune homme qui rapporte qu'il a un frère handicapé à cause du vaccin etc. : des expériences directes et très personnelles). Pensons également aux discours médiatiques des hommes politiques (notamment sur les réseaux sociaux : Twitter ou Facebook) : ce sont souvent des flashes d'expériences insignifiantes de leur vie (un dîner, un moment avec leurs enfants, le jogging...). II faut donc prendre en considération que de tels exemples ne sont pas de véritables cas de véridiction, au sens traditionnel du terme. Dans le carré de la vérité, nous ne pourrions pas placer ces cas sur l'axe de la fausseté, sur celui du mensonge ni sur celui du secret (au contraire : ici, nous sommes en présence d' « expositions », et non pas de secrets ; d'expériences directement vécues, et non pas de mensonges). Cependant, il s'agit de cas que je définirai comme " polluants », puisqu'un élément de la réalité vient jouer un rôle argumentatif erroné, en faussant les conclusions d'un argument.

Le problème est d'utiliser ces éléments de l'expérience subjective en tant que preuves d'une conclusion générale: une théorie générale (les vaccins sont mauvais), une crédibilité générale (ce politicien est sensible et donc crédible).

En bref: l'authenticité de l'expérience devient la garantie du dire, indépendamment de toute considération de contexte (le degré de généralité de la conclusion, les compétences du locuteur, le cadre du discours...) et donc audelà de toute logique véridictoire: l'axe central n'est plus la dialectique être/paraître, qui était à la base du carré de la véridiction, mais la dialectique éprouver/paraître. Le terme "réalistique » du carré (l'être) se dissout dans l'expérience. 
De ce point de vue, dans les « pathologies de l'information » de nos jours, le problème auquel nous sommes confrontés n'est pas tant le manque de professionnalisme d'un journaliste en particulier ou le manque de fiabilité d'un journal, ni encore la naïveté des lecteurs/spectateurs. La solution à ces « pathologies de l'information » ne peut pas non plus résider dans des politiques de fact-checking. Le problème ne réside pas dans la vérification des faits (toute expérience serait vérifiable) ; le problème a à voir avec la perception de ce qui est devenu pour nous un "fait », ou plutôt un fait-digne-d'importance, un fait pertinent dans la logique de l'information.

Ainsi, le problème qui, au niveau de la communication, se manifeste comme un problème de post-vérité, de fake news et d'infodémie, au niveau des systèmes de signification est un problème de logique de "pertinentisation ». Les codes qui régissent la relation entre les textes et la réalité sont des codes qui trouvent souvent leur critère dans un principe d'expérience qui délégitimise la question de la vérité. Le point crucial n'est pas que l'on raconte aujourd'hui des mensonges; le point crucial est que l'on produise des discours et des textes qui neutralisent la logique de la véridiction, en passant par le domaine de l'authentification.

Tandis que la véridiction était fondée sur l'articulation entre l'être et le paraître, ce que j'appelle l'authentification est fondé sur la dialectique entre l'être et le vécu, sur ce que vous éprouvez personnellement. Ce qui est éprouvé et ressenti définit un axe d'authenticité qui légitime tout discours. Ainsi, selon la logique traditionnelle de la véridiction le critère du dire repose sur la relation entre la manifestation sémiotique (discours, textes, signes, images... toute forme d'« externalisation » sémiotique) et la réalité ; en revanche, dans la logique de l'authentification le critère du dire repose sur la relation entre l'expérience et la réalité.

\section{Une culture super-textualisée}

Nous sommes donc face à un problème de relation avec les signes, c'està-dire à une question relative à l'attitude d'une certaine sémiosphère (voir Lotman 1985) envers ses signes. À cet égard, Lotman (1978) parlait de cultures textualisées et de cultures grammaticalisées: d'une part, des cultures qui valorisent les textes, les manifestations sémiotiques, et qui définissent ensuite leurs propres critères de structuration en les extrapolant à partir de là, et d'autre part des cultures qui valorisent les règles pour définir après les manifestations sémiotiques possibles. Je crois que la sémiosphère contemporaine, caractérisée par la post-vérité, représente une forme extrême de culture textualisée. Non seulement la priorité est donnée à la production sémiotique en elle-même, c'està-dire à l'expressivité plutôt qu'à la justesse et à l'exactitude (et, de ce fait, toute production sémiotique a une valeur en elle-même), mais cette expressivité ne 
définit pas de codes, de règles, de critères de vérité propres. Au contraire, elle se propose comme une dévaluation, un dépassement des critères de vérité au profit d'un critère d'authenticité plus subjectif et transitoire. Si la vérité aspire à la neutralité autosuffisante, à un statut supra-personnel qui conduit à un accord partagé, l'authenticité relance la subjectivité et conduit, le cas échéant, à la projection, à la synchronisation, mais pas à des codes supra-personnels.

Je crois que si nous ne parvenons pas à mettre l'accent sur cet aspect - sur cette délégitimation du critère de vérité au profit d'une pure expressivité discursive -, il sera difficile de comprendre pourquoi une série d'énoncés discordants ne sont pas perçus comme problématiques par les personnes qui les entendent. Le problème, dans notre monde de post-vérité et de fake news, n'est pas le fait que des erreurs soient commises, que l'information ne soit pas exacte, que des versions tendancieuses de la réalité soient développées à des fins de manipulation: ces phénomènes ont toujours existé. Le problème est que tout cela n'est plus perçu comme problématique ni encore comme inacceptable. II me semble que les sujets qui produisent des textes de ce type (avec des erreurs, des manipulations, des inexactitudes) préservent leur droit de parler, au nom d'une authenticité qui les « sauve ».

À ce stade, il convient de se pencher sur l'autre terme que j'ai mentionné au début de cette réflexion et que je trouve très utile pour mieux comprendre le monde de l'information qui nous entoure : celui de régime (dans le sens de Michel Foucault, qui parle de régime discursif) ${ }^{2}$.

D’après Foucault (1969), les statuts de vérité des discours reposent sur un ensemble (dont ils dépendent) d'institutions, de techniques, de politiques (au sens large) historiques - et donc modifiables - qui n'ont rien d'individuel car ces éléments se définissent au niveau social.

Les vérités ne sont pas absolues, elles ne sont pas intemporelles et ne répondent pas à une objectivité : à chaque fois que nous parlons d'objectivité, nous prétendons garantir quelque chose que nous ne pouvons pas garantir. Les vérités dépendent des critères de vérité que nous définissons culturellement.

Ce que notre époque de post-vérité met en évidence, c’est que la logique de la vérité, la manière dont nous attribuons ou reconnaissons le statut de vérité aux discours, a changé. Ce n'est pas qu'aujourd'hui la vérité n'a plus d'importance (comme on le prétend parfois face à la propagation du phénomène des fake news) ; nous affirmons et multiplions continuellement les prétentions à la vérité (pensons au domaine thérapeutique : l'autorité des institutions médicales a été flanquée de mille voix alternatives et communes, qui revendiquent leur propre vérité). En revanche, ce qui est certain, c'est que pour prétendre que quelque

${ }^{2}$ En général, sur l'utilité de l'héritage foucauldien pour la sémiotique de la culture, j'ai écrit dans Lorusso 2015, chap. 4, Lorusso 2019 et Lorusso 2020. 
chose soit vrai, pour attribuer le statut de vérité à quelque chose, nous nous appuyons sur des critères différents de ceux considérés par le passé.

Dans cette dynamique, comme je l'ai déjà souligné, le rôle des médias a été et reste fondamental. Avant même la vie " onlife » (où les expériences concrètes - the life - et les expériences en ligne - on line - s'entremêlent, comme l'a observé le philosophe Luciano Floridi, 2017), la télévision a façonné notre perception de la réalité.

En effet, ce à quoi nous sommes confrontés aujourd'hui à cause des médias est à mon avis un régime "confusionnel », un régime de réalité dans lequel différents plans (réalité, fiction, expérience, simulation, imagination...) se confondent et s'entremêlent jusqu'à la non-différenciation, avec des répercussions inévitables sur notre perception de la vérité.

Le résultat de tout cela est une forme de culture dans laquelle les logiques d'authentification, de pertinence, et donc de signification, changent profondément.

Les catégories de la sémiotique de la culture que j’ai évoquées ici nous permettent de voir que la post-vérité et les fake news ne sont pas une pure et simple pathologie contemporaine de l'information, à affronter uniquement au moyen de la dénonciation et de la répression (i.e. : aller trouver les erreurs et les corriger, dans un cadre conceptuel atomistique et de vérité-correspondance selon lequel la vérité doit être vérifiée sur chaque concept atomistique énoncé, et doit correspondre à la réalité). Au contraire, ces phénomènes nous parlent d'une sémiosphère qui a changé ou qui est en train de changer les attitudes sémiotiques envers les signes, et dans laquelle les règles de signification et de légitimation font émerger de nouveaux paradigmes de validation. Ces paradigmes contournent le critère traditionnel de vérité et sapent l'idée, propre aux Lumières, d'un accord in the long run autour de vérités partagées.

Nous assistons, me semble-t-il, à un changement culturel profond (corrélatif à un changement également profond des technologies de communication et d'expression de soi). La sémiotique de la culture pourra nous aider si, dans ses analyses des dynamiques informationnelles, elle réussit à passer de la communication aux codes de la signification.

\section{Les tâches de l'analyse sémiotique}

Pour conclure, je proposerai une réflexion sur les avantages d'une sémiotique de la culture, ainsi que sur ses tâches.

Je ne pense pas que la sémiotique de la culture doive être définie en termes d'objets particuliers : elle peut s'exercer, toujours avec la même légitimité, sur des textes littéraires, sur des discours publicitaires, sur des chefs-d'œuvre de l'histoire 
de l'art, sur des émissions télévisées ou sur des rites et des formes de vie. Ce qui la définit - et ce qui la rend précieuse dans l'économie globale du champ sémiotique, où d'autres spécialisations trouvent leur place -, c'est l'attention portée aux logiques culturelles qui sous-tendent l'objet analysé. Qu'est-ce que j'entends par " logique culturelle »? Je me réfère par là - comme je l'ai suggéré au début - aux critères de pertinence et de valorisation du réel, ce qui légitime la signification comme appropriée, conforme et significative.

Autrement dit, avant même d'analyser les valeurs, les narrations et les textes propres à une certaine sémiosphère, il est essentiel d'identifier les critères de légitimité que la sémiose possède dans cette sémiosphère : c'est ainsi que Lotman (1978, op. cit) distinguait la logique classique de la logique baroque et Omar Calabrese (1992) parlait de néo-baroque. C'est en fonction du critère de légitimation des signes que l'on pourra analyser le rôle et la fonction que certains signes, textes et discours ont au sein d'une sémiosphère spécifique.

Dans une sémiosphère dominée par un critère d'imitation et de répétition, toute manifestation d'originalité sera délégitimée; en revanche, dans une sémiosphère dominée par des critères d'expressivité, toutes les manifestations créatives deviendront centrales. De ce point de vue, la distinction lotmanienne entre cultures grammaticalisées et cultures textualisées a probablement besoin d'être élargie. L'alternative culturelle n'est pas seulement entre règle et expression, grammaire et texte, car à côté des cultures de règles et des cultures de textes peuvent également se développer des cultures d'imitation - où l'imitation n'est pas seulement l'application de la règle à des cas potentiellement différents, mais la répétition de cas analogues et la dissimulation de la variabilité, comme au Moyen Âge, où l'on comptait sur l'imitation des maîtres, en reposant "sur les épaules de géants » -; des cultures de transgression - où la transgression ne concerne pas seulement la créativité, mais implique le dépassement (quoique répétitif et peu créatif) des règles - ; des cultures de l'authenticité - où ce qui est authentique, vécu, est alors fiable, comme nous l'avons vu au sujet de l'information contemporaine -, etc. Bref, outre les Codes (les règles) et les Livres (sacrés) - qui sont les deux modèles sur lesquels Lotman raisonne -, il existe des Manuels (des instructions, qui sont des règles sui generis, et certainement pas des lois générales), des Journaux intimes (d'expériences vécues), des Manifestes (d'intention, qui aspirent à devenir des règles, mais qui sont encore de l'expressivité pure...), etc.: on a là une gamme complexe et graduée de modèles pour définir notre orientation sémiotique dans le monde.

Le cas des pathologies de l'information contemporaines nous confronte à un phénomène intéressant pour la sémiotique de la culture : il met en évidence la manière dont le " statut de vérité » sur lequel l'information devrait reposer a changé et, par conséquent, la façon dont les critères de légitimation de la sémiose ont également subi des modifications. 
Le problème devient alors celui de vérifier - dans l'analyse d'un journal, d'une émission télévisée, d'un réseau social - quelles sont les « preuves » que les sujets apportent à leurs discours, sur quoi et comment ils construisent la légitimité de ces derniers, c'est-à-dire: quel genre d'informateurs et d'observateurs mettent-ils en scène? quelles modalités épistémiques privilégient-ils; quelles instances de destination, avec quelle aspectualité ? L'analyse de la culture ne peut que partir de l'analyse textuelle ; lorsque le texte est analysé, elle doit le comparer à d'autres textes afin de " mettre en série » des phénomènes pour saisir leurs corrélations et leurs dominances (toujours selon Foucault, 1969, p. 18-19).

II me semble qu'aujourd'hui le phénomène de la post-vérité peut être « mis en série » avec d'autres formes de vie dans lesquelles un principe « confusionnel » plutôt que distinctif semble dominer : s'il y a confusion (ou superposition et hybridation) entre le vrai et le faux, il y a confusion aussi entre la vérité et la fiction, entre la fiction et la non-fiction, entre le divertissement et la politique, entre la promotion commerciale et la promotion éthique, entre la séduction et la dénonciation...

Afin d'illustrer mes propos, voici des images issues des médias de notre temps :

Figure 1 : Publicité de mode pour Amazon Fashion 2015 avec le mannequin et actrice Suki Waterhouse.

\section{Amazon Fashion}

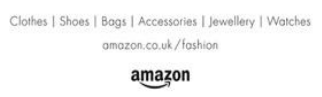

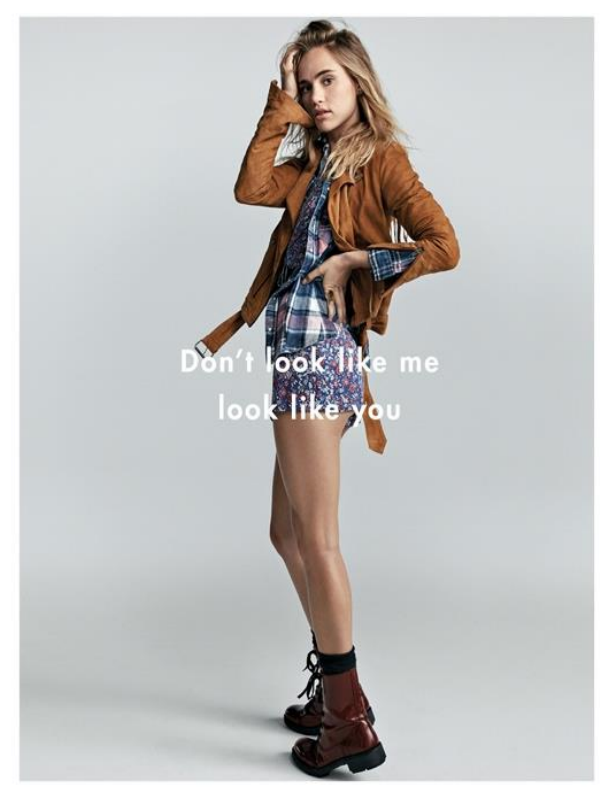


Figure 2 : Photographie utilisée par Donald Trump pendant sa première campagne présidentielle, qui fait référence à l'émission télévisée qu'il a dirigée, "The Apprentice".

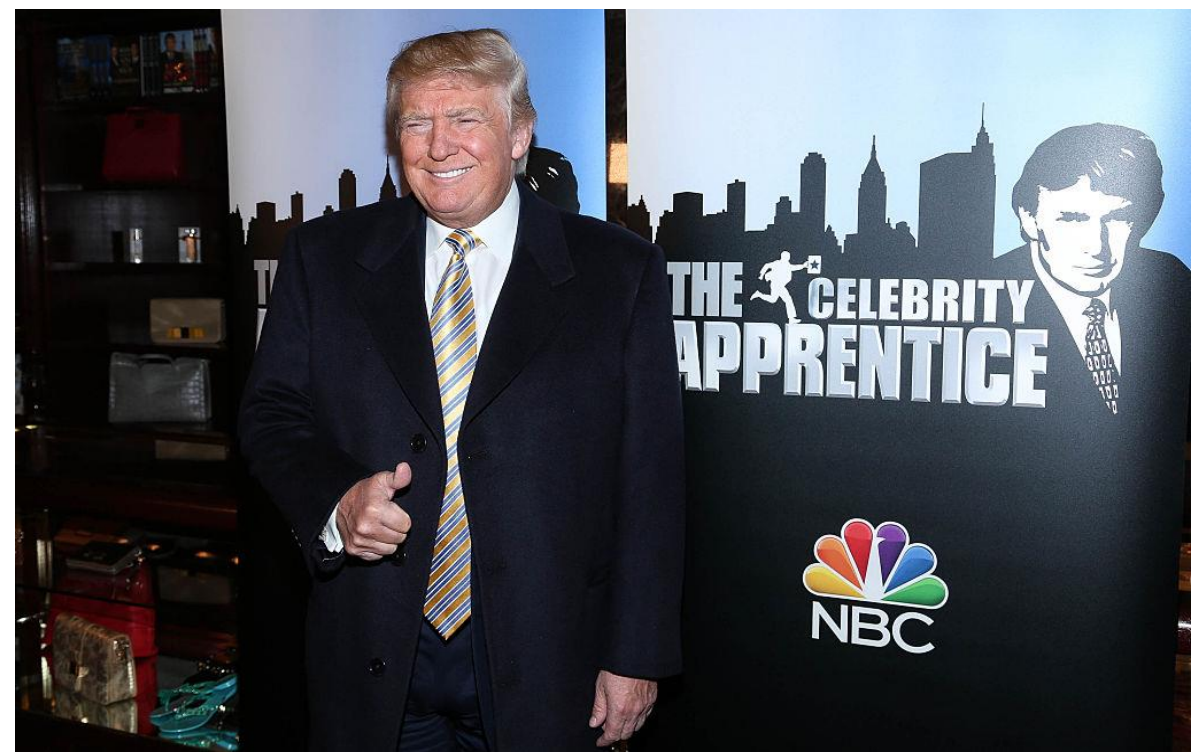

Figure 3 : Tweet du leader du parti italien populiste et de droite « Lega » - Matteo Salvini - où il se vante d'acheter seulement des fruits et des légumes italiens.

$12: 49$

$. .11 \div$

$<$

Tweet

Matteo Salvini $\otimes$

@matteosalvinimi

Negozietto fra Marconi e Magliana, si comprano frutta e verdura, sempre italiane U1, per il "cenone" intimo di stasera : $^{\circ}$

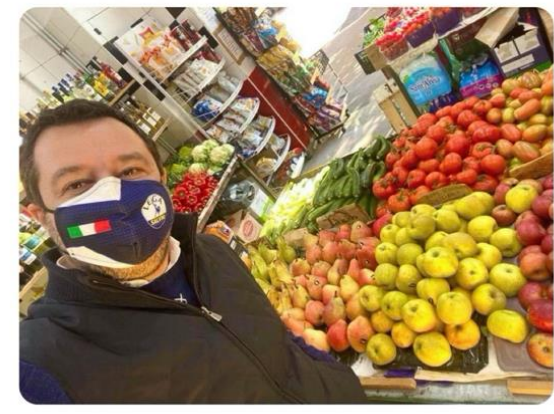

13:37 $\cdot 31 / 12 / 20 \cdot$ Twitter for iPhone

70 Retweet 8 Tweet di citazione $\mathbf{8 2 9}$ Mi piace

Twitta una risposta

10 Q 
Figure 4 : Tweet devenu viral sur les réseaux sociaux italiens en juillet 2018 , qui représente une migrante (Josefa) arrivée avec un bateau en Italie qui, malgré son état, a les ongles vernis.

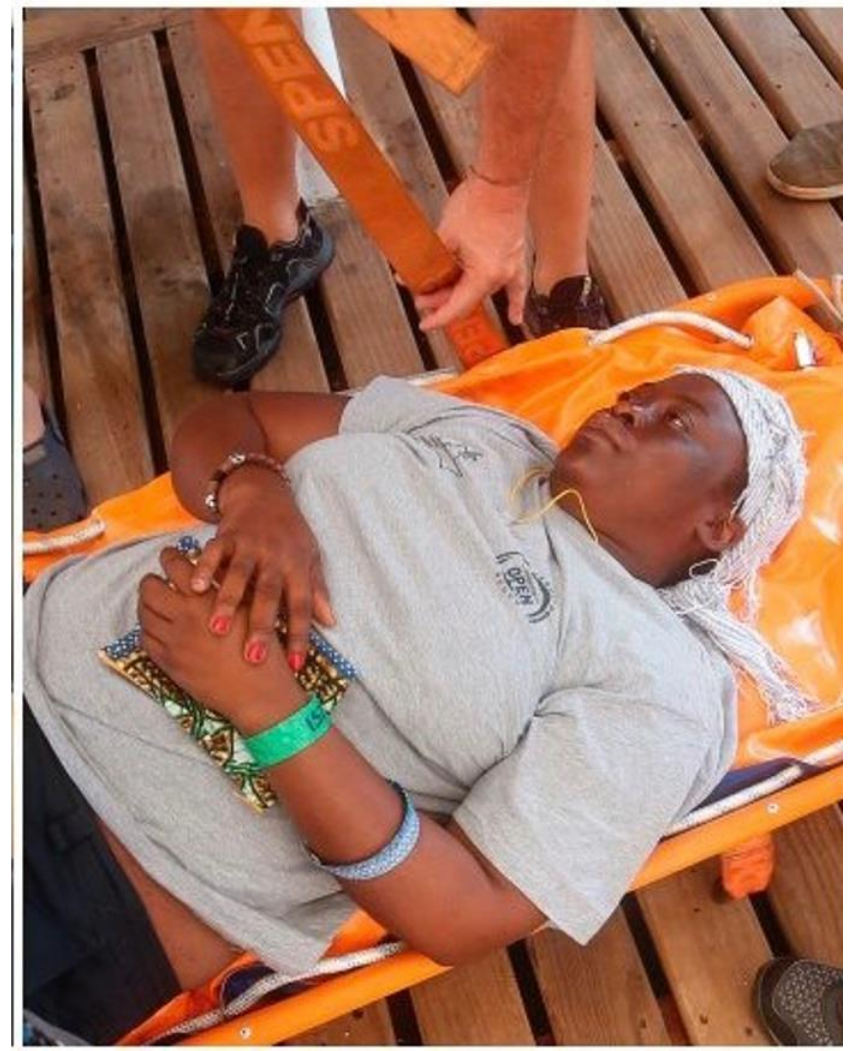

Que représentent ces images?

I. Une photographie de mode qui exprime une invitation éthique à être soi-même (en contradiction avec la logique imitative qui régit la mode).

II. Une photo de l'ancien Président américain qui rappelle son succès en tant qu'animateur d'une émission de télévision: "The Apprentice ».

III. Un twitter du leader de la droite italienne, Matteo Salvini, qui utilise le cliché le plus banal de sa journée (le shopping) pour réitérer un message de valorisation de l'italianité (au détriment de ce qui est étranger, et notamment l'immigration, selon son slogan «les |taliens avant tout! »).

IV. La photographie d'une migrante arrivée en Italie sur un bateau montrant ses ongles vernis (la photo a fait l'objet d'une forte polémique en Italie entre ceux qui prétendaient qu'il s'agissait d'une photo retouchée, ceux qui utilisaient cette photo pour dire que, si 
les migrants ont le temps de se vernir les ongles, ils ne vivent pas si mal que cela, et ceux qui admettaient, de leur côté, qu'il s'agissait d'une photo authentique, mais en expliquant sa genèse).

Mais pourquoi est-ce que je « mets en série » ces images?

Parce qu'il me semble que tous ces cas expriment la logique confusionnelle dont je parle, qui suspend les critères de vérité. Toutes ces photos expriment une vérité : elles ne sont pas fausses, elles ne peuvent être niées par aucune opération de vérification des faits.

Cependant, au sein du circuit communicatif dans lequel elles circulent, le plus pertinent est, à mes yeux, la « preuve d'authenticité » qu'elles prétendent fournir.

La première image nous invite explicitement à être nous-même, à être authentiques (bien qu'il s'agisse d'un message promotionnel). La deuxième met au même niveau les rôles médiatiques et les rôles politiques de Trump, au nom du même sujet d'expérience qui a vraiment fait les deux (ainsi, le succès d'une expérience se projette sur l'autre type d'expérience). Le tweet de Salvini montre une expérience quotidienne et témoigne de la façon dont Salvini, dans sa vie quotidienne, fait des choix pro-italiens. La photo de la migrante capture un élément corporel - le détail féminin du vernis pour " prouver » que les migrants ne sont pas aussi désespérés qu'ils le disent.

Toutes les photos dépassent les limites de leur genre discursif : la publicité de mode devient un discours édifiant ; le discours politique se mélange avec celui du spectacle télévisuel; un tweet apparemment privé se transforme en un message politique ; une photo documentaire devient une preuve pour développer un discours politique et polémique.

Toutes les photos adhèrent à la vérité, à un certain type de vérité, en la déplaçant à un autre niveau.

Tel est, selon moi, le jeu sémiotique typique d'aujourd'hui.

Il est inutile de se référer au carré de la vérité dans tous ces cas ; pour de nombreux phénomènes actuels, il n'est pas pertinent.

II est plus utile de penser à la logique d'hybridation, d'authentification, de projection, que le régime confusionnel dans lequel nous sommes plongés place devant nous: une sorte d'exaspération du principe néo-baroque d'hybridation dans lequel les jeux optiques se multiplient et la réalité se réfracte. 


\section{Références}

CALABRESE, Omar. L'età neobarocca. Roma-Bari: Laterza, 1992.

ECO, Umberto. Trattato di semiotica generale. Milano: Bompiani, 1975 (nuova ed. Milano: La nave di Teseo, 2016).

FLORIDI, Luciano. La quarta rivoluzione. Come l'infosfera sta trasformando il mondo. Milano: Raffaello Cortina, 2017.

FOUCAULT, Michel. L'archéologie du savoir. Paris : Gallimard, 1969.

LORUSSO, Anna Maria. Cultural Semiotics. For a cultural perspective in Semiotics. New York: Palgrave-Macmillan, 2015

LORUSSO, Anna Maria. Post-verità. Fra reality TV, social media e storytelling. Roma-Bari: Laterza, 2018.

LORUSSO, Anna Maria. Sémiotique et culture. In: Amir Biglari (éd.), La sémiotique et son autre. Paris : Kimé, 2019.

LORUSSO, Anna Maria. Between truth, legitimacy and legality in the Post-truth Era. International Journal for the Semiotics of Law, vol. 33, issue 4, 2020, p. 1-13.

LOTMAN, Juri. On the Semiotic Mechanism of Culture. New Literary History, vol. 9, No. 2, Soviet Semiotics and Criticism: An Anthology (Winter, 1978), p. 211-232, The Johns Hopkins University Press.

LOTMAN, Juri. La semiosfera. Venezia: Marsilio, 1980

LOTMAN, Juri; USPENSKIJ, Boris (éd.). Travaux sur les systèmes de signes. Bruxelles :

Complexe, 1976 


\title{
क0) Logic of information and Semiotics of culture
}

\author{
๑ LORUSSO, Anna Maria
}

\begin{abstract}
What is post-truth? What are fake news? A pure pathology of information, or a phenomenon that reveals a different logic of social signification? Thanks to the tools of the semiotics of culture (in particular the semiotics of Umberto Eco and Juri Lotman), the text highlights how the criterion of truth of the discourses has radically changed (the "regime of truth", as Foucault would say) and how a "confusive" regime has emerged that focuses on a criterion of authenticity based on experience, rather than a criterion of truth based on verification.
\end{abstract}

Keywords: information; post-truth; fake news; realism; authenticity.

\section{Como citar este artigo}

LORUSSO, Anna Maria. Logique de l'information et sémiotique de la culture. Estudos Semióticos [online], volume 17, número 2. Dossiê temático: "A Semiótica e a cultura". São Paulo, agosto de 2021. p. 307-320. Disponível em: <www.revistas.usp.br/esse>. Acesso em: $\mathrm{dia} / \mathrm{mês} / \mathrm{ano}$.

\section{How to cite this paper}

LORUSSO, Anna Maria. Logique de l'information et sémiotique de la culture. Estudos Semióticos [online], vol. 17.2. Thematic issue: "Semiotics and culture". São Paulo, august 2021. p. 307-320. Retrieved from: <www.revistas.usp.br/esse>. Accessed: month/day/year.

Data de recebimento do artigo: 23/01/2021.

Data de aprovação do artigo: 15/03/2021. 